\title{
Role of Soluble Triggering Receptors Expressed on Myeloid Cells - 1 and 25-Hydroxy Vitamin D as Early Predictors of Neonatal Ventilator Associated Pneumonia
}

\author{
Mai Rabie El-Sheikh \\ Tanta University Faculty of Medicine \\ Heba Said Elmahdy \\ Tanta University Faculty of Medicine \\ Mohammed Abd Ellatif Nassar \\ Tanta University Faculty of Medicine \\ Mohamed Hosni Fouda \\ Tanta University Faculty of Medicine \\ Ashraf ibrahim ( $\square$ ashraf.abohamama@med.tanta.edu.eg ) \\ Tanta University Faculty of Medicine https://orcid.org/0000-0002-5241-6695
}

\section{Research Article}

Keywords: Neonates, Ventilator associated pneumonia, sTREM-1, Vitamin D

Posted Date: February 1st, 2022

DOI: https://doi.org/10.21203/rs.3.rs-1281192/v1

License: (c) (i) This work is licensed under a Creative Commons Attribution 4.0 International License.

Read Full License 


\section{Abstract}

Ventilator associated pneumonia (VAP) is considered one of the most nosocomial pneumoniae in ventilated neonates. Yet, its diagnosis is very difficult due to non-specific clinical parameters and the lack of sensitive biomarker. The main objective of this study was to assess comparison between soluble triggering receptors expressed on myeloid cells -1 (sTREM-1) and 25-hydroxy vitamin D as early predictors of neonatal VAP. This prospective cohort study included 85 ventilated neonates who were divided into two groups; VAP group $(n=33)$ and non-VAP group $(n=52)$. sTREM-1 level in the endotracheal aspirate (ETA) and serum 25-Hydroxy vitamin D were measured on the third and seventh days following mechanical ventilation. sTREM- 1 cutoff value of $>0.46$ and $>0.44 \mathrm{ng} / \mathrm{ml}$ at 3 and 7 days had a sensitivity of $93.94 \%$ and $96.97 \%$, a specificity of $92.31 \%$ and $100 \%$, and area under the receiver operating characteristic curve (AUC) of 0.963 and 0.993 respectively to predict the development of neonatal VAP. A cutoff value of $\leq 17.5 \mathrm{ng} / \mathrm{ml}$ for serum 25-Hydroxy vitamin $\mathrm{D}$ at 3 and 7 days had a sensitivity of $90.91 \%$ and $81.82 \%$, a specificity of $75 \%$ and $78.85 \%$, and AUC of 0.877 and 0.939 respectively.

Conclusion: Both sTREM-1 in ETA and serum 25-Hydroxy vitamin D could be used as early predictors of neonatal VAP, but sTREM-1 appears to be more useful.

\section{What Is Known}

- Ventilator associated pneumonia (VAP) is one of the most nosocomial pneumoniae in ventilated patients and is associated with prolonged hospitalization and higher mortality.

- There are no specific clinical parameters for VAP, and no specific biomarker has been identified for neonatal VAP.

\section{What is New:}

- Both sTREM-1 in the endotracheal aspirate (ETA) and serum 25-Hydroxy vitamin D could be used as early predictors of neonatal VAP.

- sTREM-1 appears to be more useful as it showed higher sensitivity and specificity.

\section{Introduction}

Mechanical ventilation (MV) is considered a crucial component of the modern neonatal care, but it is frequently associated with an increased risk of VAP which is one of the most nosocomial pneumoniae in ventilated patients, and it usually develops after 48 hours of ventilation. VAP is associated with prolonged hospitalization and higher mortality [1, 2].

The diagnosis of VAP is difficult, for many reasons, first, there is no specific clinical parameters for VAP, and depending on them usually leads to overestimation and antibiotic misuse [3]. Second, no specific 
biomarker has been identified for neonatal VAP [4].

Some biomarkers are considered adjunctive tools for the diagnosis of pneumonia including C-reactive protein (CRP), leucocytic count, immunoglobulins, and other pro-inflammatory cytokines [4]. However, all of these markers are not specific for VAP and can increase in any other inflammatory conditions [5].

sTREM represents a crucial element of the innate immunity against infections [6]. During acute inflammatory response, TREM- 1 is expressed on the surface of neutrophils, macrophages, and monocytes [7]. Elevated sTREM-1 levels have been detected in bronchoalveolar lavage fluids of patients with pneumonia, in the exhaled breath condensate in VAP patients, and in patients with sepsis [8].

Vitamin $\mathrm{D}$ has a vital role in immune system function and regulation. There is an association with respiratory infections and inadequate serum vitamin $D$ in children [9-11].

Due to the paucity of studies and ongoing need for more specific biomarkers of neonatal VAP, we conducted the current study to evaluate the diagnostic value of sTREM-1 and 25-Hydroxy vitamin D as early predictors of neonatal VAP.

\section{Methods}

This prospective cohort study was conducted on 85 neonates who required MV within 48h of birth and needed it for more than 7 days, admitted in the NICU of Tanta University Hospitals from September 2020 to September 2021. Neonates with infectious lung diseases before intubation, multiple congenital anomalies and multiple organ failure were excluded.

The included cases were divided into two groups: VAP group and non-VAP group. VAP definition proposed by Centers for Disease Control and Prevention (CDC) and the National Healthcare Safety Network (NHSN) was used $[1,12]$.

All patients were ventilated by orotracheal tube using Neumovent ventilator (Neumovent Graphnet Ts, Tecme Corporation, USA) with heated humidification system. One set of disposable ventilator circuit was used for one patient, and open suction was the method used. No topical oral antibiotic or antiseptic solutions were used for cleaning of the mouth.

Serial chest radiographs were ordered for all cases on $0,1,3,5$ and 7 days after MV. Complete blood count, CRP, serum 25-Hydroxy vitamin D, endotracheal aspirate WBCs and STREM-1 were recorded on the 3rd and 7th days of MV.

\section{Endotracheal aspirate sTREM-1 level}

Two ETA for sTREM-1 were taken for each patient. The procedure was performed using an end hole suction catheter filled with sterile water and connected to a low-pressure suction apparatus. 0.5-1 ml sterile water was injected in the tube after its disconnection from the ventilator circuit, via a sterile 
disposable syringe. The suction catheter was immediately inserted into the ETT, for suction of the fluid coming back from the lower airways. The obtained fluid was then collected in a sterile container and immediately sent for microbiological examination. The sample was divided into two parts, the first portion was centrifuged for 15 minutes and stored at refrigerator at $-20^{\circ} \mathrm{C}$ for calculation of STREM- 1 levels and the other one was used for microbiological examination and culture. sTREM-1 level was assessed using the commercially available ELIA EIAab kit (Wuhan, China).

\section{Serum 25-Hydroxy vitamin D level}

Serum 25-Hydroxy vitamin D level was measured by ELISA technique. Venous blood was collected from a sterile venipuncture and put in tubes labeled with the patient's name. All tubes were centrifuged after it allowed to clot at room temperature for separation of serum from whole blood. The serum was drowned and stored at $2: 8^{\circ} \mathrm{C}$.

\section{Statistical analysis}

Using MedCalc ${ }^{\circledR}$ program version 20.009 (MedCalc Software, Ostend, Belgium). The sample size was calculated by the following criteria: 0.05 alpha error, $80 \%$ power of the study. AUC for sTERM to predict neonatal VAP was expected to be 0.902 and vitamin D was 0.75 . Therefore, 85 cases were recruited.

The collected data were statistically analyzed using Statistical Package for the Social Sciences (SPSS 26.0, IBM/SPSS Inc., Chicago, IL) software. Categorical data were expressed as frequencies and percentages (\%) and compared by Chi-Square test (or Fisher's exact test). In the quantitative data, mean and standard deviations (SD) (for normally distributed data) and median and inter-quartile range (IQR) (for abnormally distributed data) were used. To compare two groups with normally distributed quantitative variables, independent-Samples t-test was used and Mann-Whitney $U$ test was used if the data were abnormally distributed. Pearson's correlation coefficient was used to assess the correlation between various parameters. ROC curve was used estimate the cut-off and the diagnostic ability of STREM-1 and vitamin D in the prediction of VAP. A two-tailed $P$ values $<0.05$ were considered significant.

\section{Results}

Eighty five neonates included in this study, VAP group included 33 neonates, while non-VAP group included the remaining 52 neonates who did not develop VAP in the $1^{\text {st }}$ week of MV. Baseline criteria of both groups were shown in Table 1, caesarean section, duration of MV, oligohydramnios, $\mathrm{H}_{2}$ blockers use, central venous catheter insertion, sedation and total parenteral nutrition were significantly higher in VAP group compared to non-VAP group,

Regarding laboratory parameters, WBCs count, immature to total (I/T) ratio, CRP, WBCs count in the endotracheal aspirate were significantly higher in the VAP group on both 3-and 7-day. While platelet count showed significant elevation in the non-VAP group on both 3-and 7-day. Moreover, the VAP group expressed significantly higher CRP levels. 
25-Hydroxy vitamin D was significantly lower in VAP group on both 3-and 7-day. On the other hand STREM-1 levels in the ETA showed a significant elevation in VAP cases on both 3 -and 7-day $(p<0.001)$ (Table 2).

STREM-1 had significant positive correlation with I/T ratio, leucocytic count in the endotracheal tube aspirate and WBCs. Conversely, significant negative correlation was noted between 25-Hydroxy vitamin D and these parameters. Also, sTREM- 1 had a significant negative correlation with 25 -Hydroxy vitamin D (Table 3).

sTREM-1 in ETA cutoff values of $>0.46$ and $>0.44 \mathrm{ng} / \mathrm{ml}$ at 3 and 7 days could predict the development of neonatal VAP with a sensitivity of $93.94 \%$ and $96.97 \%$, a specificity of $92.31 \%$ and $100 \%$, and an area under the curve/receiver operator characteristic of 0.963 and 0.993 respectively. Cutoff values of $\leq 17.5$ $\mathrm{ng} / \mathrm{ml}$ for serum 25-Hydroxy vitamin $D$ at both 3 and 7 days could also predict the development of neonatal VAP with a sensitivity of $90.91 \%$ and $81.82 \%$, a specificity of $75 \%$ and $78.85 \%$, and with an area under the curve/receiver operator characteristic of 0.877 and 0.939 respectively (Fig. 1).

sTREM-1 in ETA had a significantly better diagnostic ability compared to serum 25 -Hydroxy vitamin $D$ at the 3rd and 7th day $(p=0.03$ and 0.009$)$ respectively.

\section{Discussion}

VAP, a common hospital-acquired infection in the neonatal intensive care units, markedly increases the treatment time and the expense of infant patients and elevates their fatality rate. In the current study; the total leucocytic count, I/T ratio and CRP levels were significantly higher in neonates who developed VAP than those without VAP. These results agree with Badr et al. [13]. Also, the total duration of MV was statistically significant higher in VAP group compared to non VAP group (19 versus 9 days respectively).

Serum 25-Hydroxy vitamin D level was significantly lower in the VAP group compared to the non-VAP group. This came in agreement with the study by El-Kassas et al. [14] who showed that neonates with pneumonia and mechanically ventilated neonates had significant lower levels of Vit. D.

As regard to STREM- 1 level, there was significant increase in STREM-1 in the ETA at both $3^{\text {rd }}$ and $7^{\text {th }}$ day of MV in VAP cases. Furthermore, sTREM-1 was positively correlated with serum WBCs, I/T ratio, leucocytic count in the ETA. However, it was negatively correlated with serum 25-Hydroxy vitamin D level. In agreement with our findings, Yang et al. [15] revealed that serum and bronchoalveolar lavage fluid STREM-1 levels were significantly higher in neonatal VAP group than in controls. A meta-analysis by Bellos et al suggested that STREM- 1 could be used as a useful biomarker for neonatal sepsis prediction [16].

Using a cut off value of $>0.46 \mathrm{ng} / \mathrm{ml}$, that marker had $93.94 \%$ sensitivity and $92.31 \%$ specificity to predict the occurrence of VAP on $3^{\text {rd }}$ day and using a cut off value $>0.44$ it had $96.97 \%$ sensitivity and $100 \%$ specificity to predict the occurrence of VAP on the $7^{\text {th }}$ day. 
On the other hand, there was significant decrease in serum 25-Hydroxy vitamin D level at both $3^{\text {rd }}$ and $7^{\text {th }}$ day of MV in VAP cases and it had significant negative correlation with leucocytic count in the endotracheal tube aspirate, total leucocytic count and I/T ratio.

A recent study conducted by Zhou and his colleagues [17] reported that STERM in the bronchoalveolar lavage-fluid had sensitivity and specificity of $100 \%$ and $93.7 \%$ respectively with AUC of 0.938 in predicting VAP in neonates regardless of extrapulmonary infections. Moreover, Zhao et al. [18] found that serum sTERM level showed a significant increase in the VAP group compared to the non-VAP one at 72 and 120 hours after ventilation.

To the best of our knowledge, there are few studies in the current literature handling the evaluation of sTREM-1 in endotracheal aspirate for neonatal VAP diagnosis and this is the first study to compare serum 25-Hydroxy vitamin D and sTREM-1 in ETA for prediction of neonatal VAP.

The current study has some limitations; it was a single center study, the included sample size was relatively small and serum level of STREM-1 should have been also assessed to decide which sample would be better for evaluation. These drawbacks should be well-covered in the upcoming studies.

\section{Conclusion}

Both sTREM-1 in ETA and serum 25-Hydroxy vitamin D could be used as early predictors of neonatal VAP, but sTREM-1 appears to be more useful.

\section{Abbreviations}

AUC, area under the receiver operating characteristic curve; CDC, Centers for Disease Control and Prevention; CRP, C-reactive protein; ETA, endotracheal aspirate; ETT, endotracheal tube; I/T ratio, Immature-to-total neutrophil ratio; MV, mechanical ventilation; NHSN, National Healthcare Safety Network; sTREM-1, Soluble Triggering Receptors Expressed on Myeloid Cells -1; VAP, Ventilator associated pneumonia.

\section{Declarations}

Acknowledgements We are thankful for all families of enrolled neonates and staff of the Neonatal Intensive Care Unit at Tanta University Hospital, Tanta, Egypt for supporting the study.

Funding: The authors declare that no funds, grants, or other support were received during the preparation of this manuscript.

Competing Interests: The authors have no relevant financial or non-financial interests to disclose.

Availability of data and material: available when required. 
Code availability: Not applicable.

Authors' Contributions: [Mai Rabie El-Sheikh], [Heba Said Elmahdy] and [Mohammed Abd Ellatif Nassar] collected, analyzed the data and approved the manuscript, [Mohamed Hosni Fouda] performed the laboratory tests, and [Ashraf Mohamed Ibrahim] performed the statistical analysis, wrote, and revised the manuscript.

Ethical approval: The study was approved from the Ethical committee of Faculty of Medicine, Tanta University. The study is in accordance with the ethical standards of institutional research committee and with the 1964 Helsinki declaration and its later amendments.

Consent to participate: Informed written consent was obtained from the guardians of the enrolled neonates.

Consent to publication: All the authors transfer, assign, or otherwise convey all copyright ownership, including any and all rights exclusively to the journal, in the event that such work is published by the journal.

\section{References}

1. Cernada M, Brugada M, Golombek S, Vento M (2014) Ventilator-Associated Pneumonia in Neonatal Patients: An Update. Neonatology 105(2):98-107. https://doi.org/10.1159/000355539

2. Goerens $A$, Lehnick $D$, Büttcher $M$, Daetwyler $K$, Fontana $M$, Genet $P$, Lurà $M$, Morgillo $D$, Pilgrim $S$, Schwendener-Scholl K, Regamey N, Neuhaus TJ, Stocker M (2018) Neonatal ventilator associated pneumonia: a quality improvement initiative focusing on antimicrobial stewardship. Front Pediatr 6:262. https://doi.org/ 10.3389/fped.2018.00262

3. Rea-Neto A, Youssef NC, Tuche F, Brunkhorst F, Ranieri VM, Reinhart K, Sakr Y (2008) Diagnosis of ventilator-associated pneumonia: a systematic review of the literature. Crit Care 12:R56. https://doi.org/10.1186/cc6877

4. Fernando SM, Tran A, Cheng W, Klompas M, Kyeremanteng K, Mehta S, English SW, Muscedere J, Cook DJ, Torres A, Ranzani OT, Fox-Robichaud AE, Alhazzani W, Munshi L, Guyatt GH, Rochwerg B (2020) Diagnosis of ventilator-associated pneumonia in critically ill adult patients-a systematic review and meta-analysis. Intensive Care Med 46(6):1170-1179. https://doi.org/10.1007/s00134020-06036-Z

5. Summah H, Qu J-M (2009) Biomarkers: A Definite Plus in Pneumonia. Mediators Inflamm. 2009:675753 https://doi.org/10.1155/2009/675753

6. El Nady HG, Kholoussi N, Sherif LS, El Baroudy NR, El Refay AS, Abdelkawy RFM (2019) Triggering Receptor Expressed on Myeloid Cells-1 (TREM-1) as a New Marker in Ventilated Children with Pneumonia. Biomed Pharmacol J 12(4). https://dx.doi.org/10.13005/bpj/1826 
7. Kouassi KT, Gunasekar P, Agrawal DK, Jadhav GP (2018) TREM-1; Is It a Pivotal Target for Cardiovascular Diseases? J Cardiovasc Dev Dis 5(3):45. https://doi.org/10.3390/jcdd5030045

8. Phua J, Koay ES, Zhang D, Tai LK, Boo XL, Lim KC, Lim TK (2006) Soluble triggering receptor expressed on myeloid cells-1 in acute respiratory infections. Eur Respir J 28:695-702. https://doi.org/10.1183/09031936.06.00005606

9. Walker VP, Modlin RL (2009) The vitamin D connection to pediatric infections and immune function. Pediatr Res 65(5 Pt 2). https://doi.org/10.1203/PDR.0b013e31819dba91. :106R-113R

10. Sibaii H, El-Zayat SR, El-Shaheed AA, Mahfouz NN, Sallam SF, El Azma MH (2016) The Hidden Function of Vitamin D. Open Access Maced J Med Sci 4(4):591-595.

https://doi.org/10.3889/oamjms.2016.134

11. Siljan WW, Holter JC, Michelsen AE, Nymo SH, Lauritzen T, Oppen K, Husebye E, Ueland T, Mollnes TE, Aukrust P, Heggelund L (2019) Inflammatory biomarkers are associated with aetiology and predict outcomes in community-acquired pneumonia: results of a 5-year follow-up cohort study. ERJ Open Res 5(1):00014-2019. https://doi.org/10.1183/23120541.00014-2019

12. Horan TC, Andrus M, Dudeck MA (2008) CDC/NHSN surveillance definition of health care-associated infection and criteria for specific types of infections in the acute care setting. Am J Infect Control 36(5):309-332. https://doi.org/10.1016/j.ajic.2008.03.002

13. Badr MA, Ali YF, Albanna EA, Beshir MR, Amr GE (2011) Ventilator associated pneumonia in criticallyill neonates admitted to neonatal intensive care unit, Zagazig University Hospitals. Iran J Pediatr 21(4):418-424

14. El-Kassas GM, El Wakeel MA, Elabd MA, Kamhawy AH, Atti MA, El-Gaffar SAA, Hanafy SK, Awadallah E (2019) Vitamin D Status in Neonatal Pulmonary Infections: Relationship to Inflammatory Indicators. Open Access Maced J Med Sci 7:3970-3974. https://doi.org/10.3889/oamjms.2019.592

15. Yang ZQ, Mai JY, Zhu ML, Xiao XM, He XX, Chen SQ, Lin ZL, Feng X (2021) Soluble Triggering Receptors Expressed on Myeloid Cells-1 as a Neonatal Ventilator-Associated Pneumonia Biomarker. Int J Gen Med 14:4529-4534. https://doi.org/10.2147/IJGM.S315987

16. Bellos I, Fitrou G, Daskalakis G, Thomakos N, Papantoniou N, Pergialiotis V (2018) Soluble TREM-1 as a predictive factor of neonatal sepsis: a meta-analysis. Inflamm Res 67(7):571-578. https://doi.org/10.1007/s00011-018-1149-4

17. Zhou J, Zhou J, Hong Y, Wang Y, Lin H, Huang L (2021) Diagnostic value of bronchoalveolar lavagefluid soluble triggering receptor expressed on myeloid cells-1 (sTREM-1) concentration for neonatal ventilator-associated pneumonia. Front Pediatr 9:664801.

https://doi.org/10.3389/fped.2021.664801

18. Zhao X, Xu L, Yang Z, Sun B, Wang Y, Li G, Feng C, Pan T, Yu T, Feng X (2020) Significance of sTREM1 in early prediction of ventilator-associated pneumonia in neonates: a single-center, prospective, observational study. BMC Infect Dis 20(1):542. https://doi.org/10.1186/s12879-020-05196-z

\section{Tables}


Table 1: Baseline criteria of the VAP group and non-VAP group 


\begin{tabular}{|c|c|c|c|}
\hline & $\begin{array}{l}\text { VAP group } \\
(n=33)\end{array}$ & $\begin{array}{l}\text { Non-VAP group } \\
(n=52)\end{array}$ & $P$ value \\
\hline Gestational age (week) & $35.85 \pm 2.25$ & $36.21 \pm 2.22$ & 0.467 \\
\hline Birth weight (Kg) & $2.36 \pm 0.47$ & $2.39 \pm 0.36$ & 0.137 \\
\hline \multicolumn{4}{|l|}{ Gender } \\
\hline Male & $19(57.58 \%)$ & $25(48.08 \%)$ & 0.393 \\
\hline Female & $14(42.42 \%)$ & 27 (51.92\%) & \\
\hline \multicolumn{4}{|l|}{ Mode of delivery } \\
\hline Normal vaginal delivery & $5(15.15 \%)$ & $14(26.92 \%)$ & $0.035^{\star}$ \\
\hline Caesarean section & $28(84.85 \%)$ & $38(73.08 \%)$ & \\
\hline \multicolumn{4}{|l|}{ Apgar score } \\
\hline 1 minute & $5.76 \pm 0.94$ & $6.04 \pm 0.93$ & 0.179 \\
\hline 5 minute & $6.58 \pm 1.80$ & $6.90 \pm 1.79$ & 0.413 \\
\hline Age at MV (days) & $1.48 \pm 0.94$ & $1.67 \pm 1.00$ & 0.391 \\
\hline Duration of MV (days) & $19.55 \pm 7.85$ & $9.37 \pm 1.67$ & $<0.001^{*}$ \\
\hline \multicolumn{4}{|l|}{$P$} \\
\hline & $4(9 \%)$ & $3(15 \%)$ & 0.423 \\
\hline & $4(9 \%)$ & $2(10 \%)$ & 0.201 \\
\hline renatal risk factors & $4(9 \%)$ & $2(10 \%)$ & 0.201 \\
\hline Anemia & $4(9 \%)$ & $0(0 \%)$ & $0.02 *$ \\
\hline Hypertension & $3(6 \%)$ & $0(0 \%)$ & 0.055 \\
\hline PROM & $3(6 \%)$ & $0(0 \%)$ & 0.055 \\
\hline Chorioamnionitis & $2(3 \%)$ & $0(0 \%)$ & 0.148 \\
\hline Preeclampsia & $2(6 \%)$ & $2(10 \%)$ & 0.639 \\
\hline \multicolumn{4}{|l|}{ Diabetes mellitus } \\
\hline \multicolumn{4}{|l|}{ Post-natal risk factors } \\
\hline Drug history (H2blockers) & $10(24 \%)$ & $0(0 \%)$ & $<0.001^{*}$ \\
\hline CVC insertion & $14(33 \%)$ & $2(4 \%)$ & $<0.001^{*}$ \\
\hline Sedation & $5(12 \%)$ & $0(0 \%)$ & 0.007 \\
\hline Total parenteral nutrition & 10 (24\%) & $2(4 \%)$ & 0.001 \\
\hline
\end{tabular}




\begin{tabular}{|llll|}
\hline Prognosis & & \\
Mortality & $4(12.12 \%)$ & $3(5.77 \%)$ & 0.423 \\
Discharge & $29(87.88 \%)$ & $49(94.23 \%)$ & 0.423 \\
\hline
\end{tabular}

VAP Ventilator associated pneumonia, $M V$ Mechanical ventilation, $P R O M$ premature rupture of membrane, $C V C$ central venous catheter * Significant as $P$ value $<0.05$

Table 2: Laboratory parameters in the studied groups 


\begin{tabular}{|c|c|c|c|}
\hline & $\begin{array}{l}\text { VAP group } \\
(n=33)\end{array}$ & $\begin{array}{l}\text { Non-VAP group } \\
(n=52)\end{array}$ & $P$ value \\
\hline \multicolumn{4}{|l|}{ Hemoglobin (gm/dl) } \\
\hline After 3 days of MV & $14.51 \pm 1.99$ & $13.76 \pm 1.87$ & 0.083 \\
\hline After 7 days of MV & $12.78 \pm 2.20$ & $13.14 \pm 1.98$ & 0.438 \\
\hline \multicolumn{4}{|l|}{ WBCs $\left(10^{3}\right.$ cells $\left./ \mathrm{dL}\right)$} \\
\hline After 3 days of MV & $12.81 \pm 3.87$ & $11.39 \pm 2.60$ & $0.047^{*}$ \\
\hline After 7 days of $\mathrm{MV}$ & $17.15 \pm 4.36$ & $14.23 \pm 5.04$ & $0.007 *$ \\
\hline \multicolumn{4}{|l|}{ Platelets $\left(10^{3}\right.$ cells $\left./ \mathrm{dl}\right)$} \\
\hline After 3 days of MV & $149.73 \pm 57.27$ & $246.19 \pm 39.44$ & $<0.001^{*}$ \\
\hline After 7 days of $\mathrm{MV}$ & $113.18 \pm 49.57$ & $243.19 \pm 39.32$ & $<0.001^{*}$ \\
\hline \multicolumn{4}{|l|}{$\mathrm{I} / \mathrm{T}$} \\
\hline After 3 days of MV & $0.149 \pm 0.029$ & $0.071 \pm 0.027$ & $<0.001^{*}$ \\
\hline After 7 days of $\mathrm{MV}$ & $0.21 \pm 0.06$ & $0.07 \pm 0.02$ & $<0.001^{\star}$ \\
\hline \multicolumn{4}{|l|}{ CRP (mg/l) } \\
\hline After 3 days of MV & $12.67 \pm 14.36$ & $6.13 \pm 6.32$ & $0.005^{\star}$ \\
\hline After 7 days of MV & $46.21 \pm 28.45$ & $6.96 \pm 3.35$ & $<0.001^{*}$ \\
\hline \multicolumn{4}{|c|}{ 25-Hydroxy vitamin $D(\mathrm{ng} / \mathrm{ml})$} \\
\hline After 3 days of MV & $13.23 \pm 3.37$ & $26.67 \pm 10.51$ & \\
\hline \multirow[t]{2}{*}{ After 7 days of MV } & $10.93 \pm 3.28$ & $23.78 \pm 9.59$ & $<0.001^{*}$ \\
\hline & & & $<0.001^{\star}$ \\
\hline \multicolumn{4}{|c|}{ Endotracheal aspirate WBCs $\left(10^{3} \mathrm{cells} / \mathrm{dl}\right)$} \\
\hline \multicolumn{4}{|l|}{ After 3 days of $\mathrm{MV}$} \\
\hline \multirow[t]{2}{*}{ After 7 days of $\mathrm{MV}$} & $31.57 \pm 23.07$ & $3.28 \pm 2.32$ & $0.005^{\star}$ \\
\hline & $83.28 \pm 45.77$ & $3.51 \pm 2.32$ & $<0.001^{*}$ \\
\hline \multicolumn{4}{|c|}{ Endotracheal aspirate sTREM-1 (ng/ml) } \\
\hline \multicolumn{4}{|l|}{ After 3 days of MV } \\
\hline \multirow[t]{2}{*}{ After 7 days of $\mathrm{MV}$} & $1.97 \pm 1.04$ & $0.31 \pm 0.13$ & $<0.001^{*}$ \\
\hline & $2.84 \pm 1.46$ & $0.32 \pm 0.08$ & $<0.001^{\star}$ \\
\hline
\end{tabular}


MV Mechanical ventilation, WBCs White blood cells, MV Mechanical ventilation, $I / T$ Immature-to-total neutrophil ratio, CRPC-reactive protein, WBCs White blood cells, sTREM-1 soluble triggering receptor expressed on myeloid cells 1 . * Significant as $\mathrm{P}$ value $<0.05$

Table 3: Correlation between sTREM-1 level and other parameters.

\begin{tabular}{|lllll|}
\hline & \multicolumn{2}{l}{ 25-Hydroxy vitamin $\mathrm{D}(\mathrm{ng} / \mathrm{ml})$} & \multicolumn{2}{l|}{ sTREM-1 $(\mathrm{ng} / \mathrm{ml})$} \\
\cline { 2 - 5 } & $R$ & $p$ & $r$ & $\boldsymbol{p}$ \\
\hline WBCs & -0.463 & $<0.001^{*}$ & 0.673 & $<0.001^{*}$ \\
\hline I/T & -0.483 & $<0.001^{*}$ & 0.731 & $<0.001$ * \\
\hline WBCS count in ETT aspirate & -0.189 & $0.014^{*}$ & 0.309 & $<0.001$ * \\
\hline 25-Hydroxy vitamin D $(\mathrm{ng} / \mathrm{ml})$ & - & - & -0.5494 & $<0.001^{*}$ \\
\hline STREM-1 $(\mathrm{ng} / \mathrm{ml})$ & -0.5494 & $<0.001^{*}$ & - & - \\
\hline
\end{tabular}

I/T Immature-to-total neutrophil ratio, ETT endotracheal tube, $r$ Pearson's correlation coefficient, sTREM-1 soluble triggering receptor expressed on myeloid cells $1, W B C s$ White blood cells

\section{Figures}
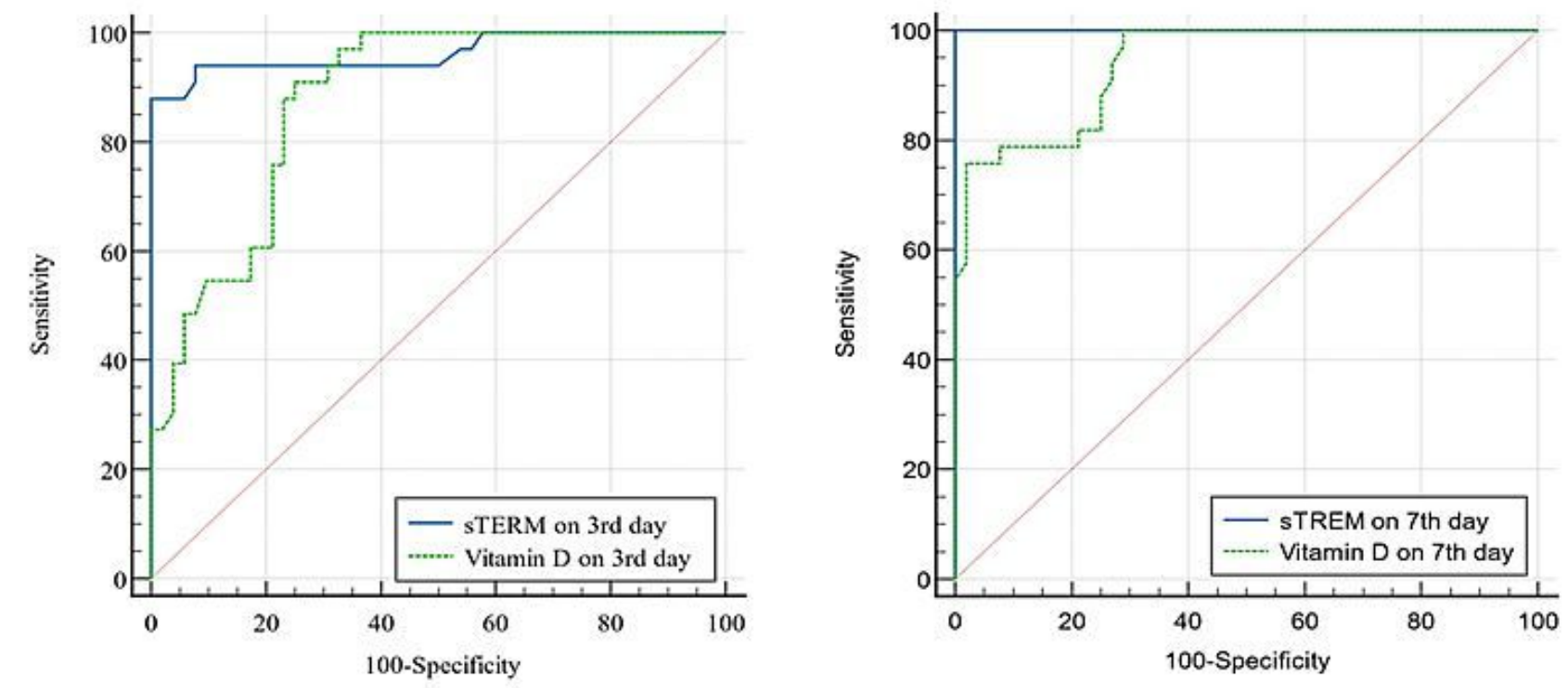

Figure 1 
ROC curve of sTREM-1 in ETA and serum 25-Hydroxy vitamin D on $3^{\text {rd }}$ day and $7^{\text {th }}$ days 